\title{
Successful endoscopic mucosal resection of a solitary extramedullary plasmacytoma in the sigmoid colon
}

A 42-year-old man was referred to our hospital as he was found to be positive for faecal occult blood at a routine checkup. Colonoscopy revealed a submucosal tumor (SMT), $9 \mathrm{~mm}$ in size, at the distal end of the sigmoid colon ( $\bullet$ Fig. 1).

Carcinoid tumor was suspected and successfully removed using endoscopic mucosal resection (EMR). Histological examinations showed diffuse infiltration of atypical plasma cells mainly in the submucosal layer ( $\bullet$ Fig. 2).

Immunophenotyping involving monoclonal antibodies to immunoglobulin revealed expression of IgG with kappa light chain restriction by the proliferating plasma cells, indicating monoclonality (๑ Fig. 3).

The lesion showed local accumulation of amyloid with Congo red staining ( Fig. 4). There was no lymphovascular invasion. Further, serum electrophoresis, bone marrow biopsy, and urinary levels of Bence Jones proteins were within normal limits. A full digestive tract survey with double-balloon enteroscopy, gallium scintigraphy, and enhanced computed tomography revealed no mass or lymphadenopathy. Therefore, a histopathological diagnosis of solitary extramedullary plasmacytoma (EMP) with a submucosal amyloid deposit $(9 \times 6 \times 5 \mathrm{~mm})$ of AL amyloidosis in the sigmoid colon was made. Following total tumor EMR, there has been no recurrence during a 36-month follow-up.

EMPs account for only $3 \%-5 \%$ of all plasma cell tumors, occurring in the upper air passage, nasal cavity, and paranasal sinuses in about $80 \%$ of cases; only $7.2 \%$ cases occur in the gastrointestinal tract [1]. The small intestine is the most common gastrointestinal site, followed by the stomach. It is extremely rare in the colon. Historically, EMPs have been treated by surgical resection because of gastrointestinal obstruction $[2,3]$, but to our knowledge, there has been no published report of an EMP being eradicated by EMR [4]. It is our impression that the endoscopic and histopathological findings of early-stage solitary EMP that we have presented here will be significant in guiding the management of similar manifestations of EMP in other patients.

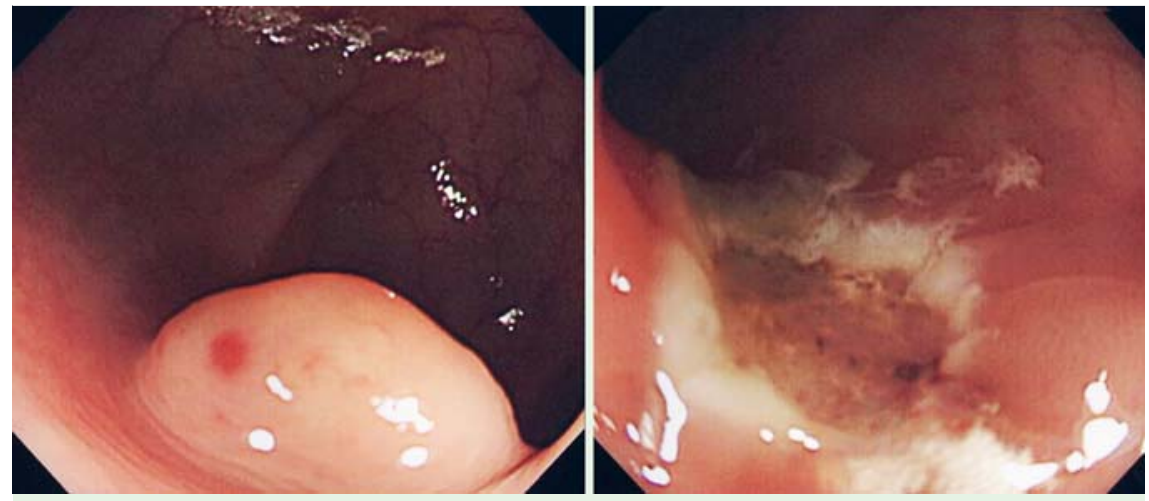

Fig. 1 A submucosal tumor $(9 \mathrm{~mm})$ in the sigmoid colon as revealed by colonoscopy and removed by endoscopic submucosal resection.
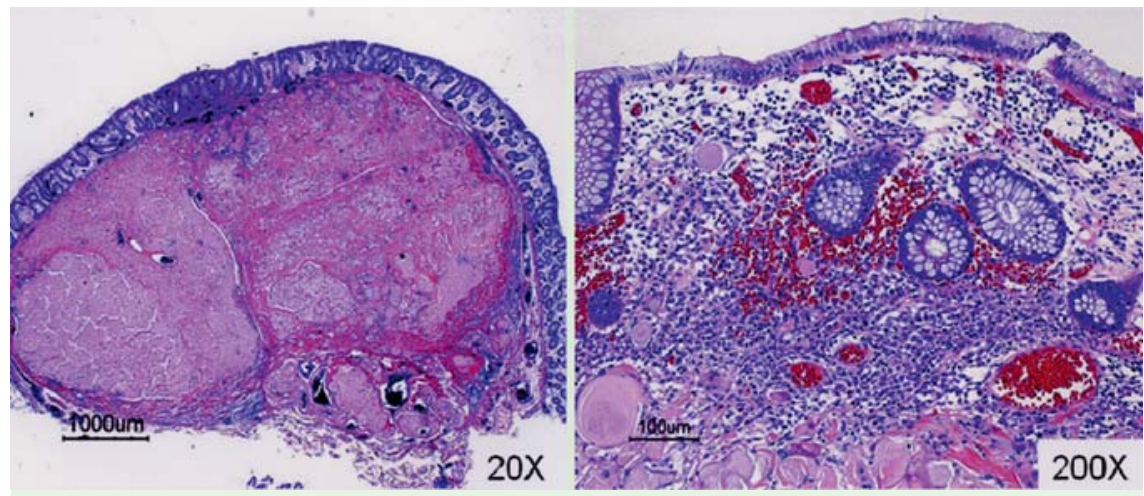

Fig. 2 Hematoxylin and eosin stained section showing diffuse infiltration of atypical plasma cells, mainly in the submucosal layer.

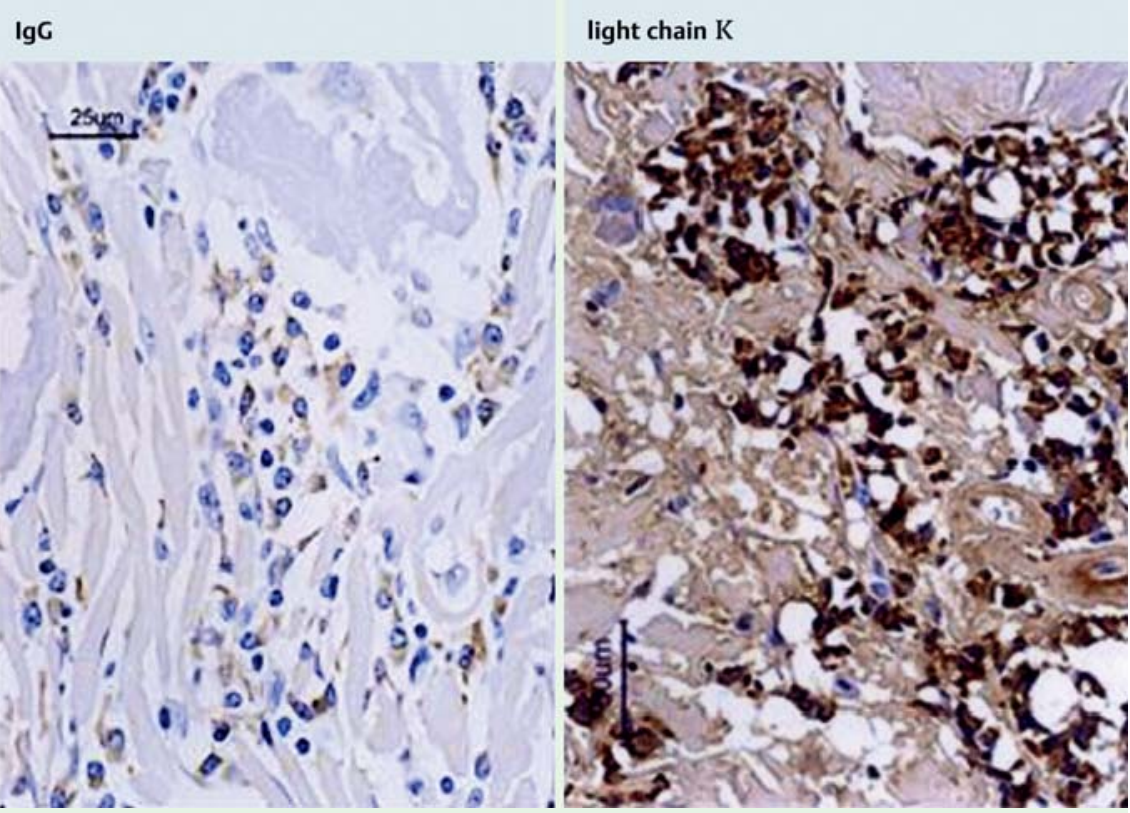

Fig. 3 Immunophenotypic analysis showing proliferating plasma cells expressing IgG with kappa light chain restriction. 

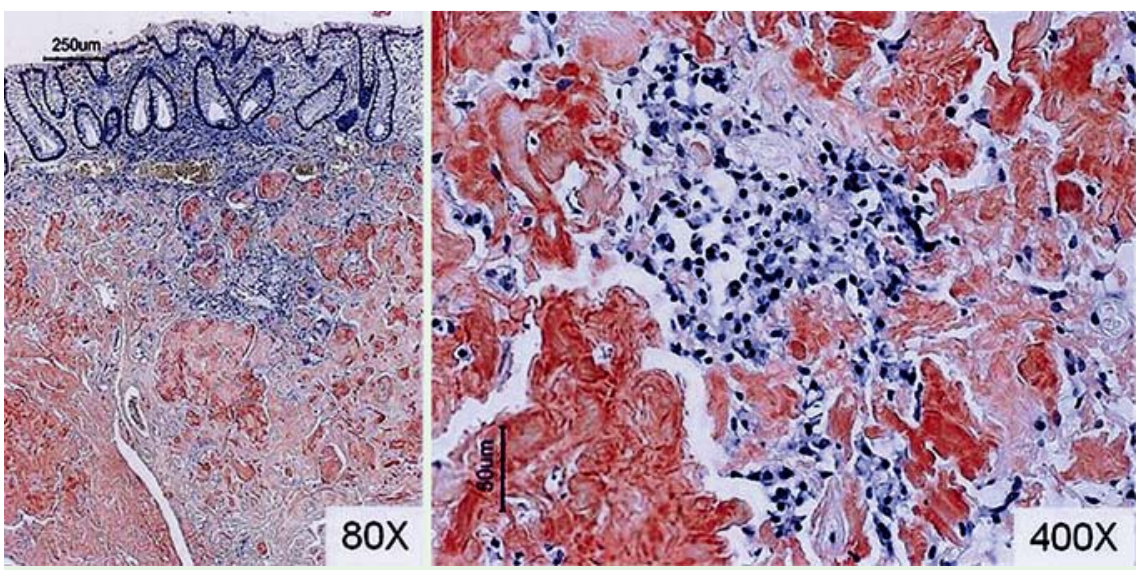

Fig. 4 Submucosal amyloid deposit revealed by Congo red staining.

Endoscopy_UCTN_Code_CCL_1AD_2AC

\section{Competing interests: None}

T. Kodani ${ }^{1}$, T. Osada ${ }^{1}$, T. Terai ${ }^{1}$,

T. Ohkusa ${ }^{2}$, T. Shibuya ${ }^{1}$, N. Sakamoto ${ }^{1}$,

K. Beppu ${ }^{1}$, J. Kato ${ }^{1}$, A. Nagahara ${ }^{1}$,

H. Watanabe ${ }^{3}$, S. Watanabe ${ }^{1}$

1 Department of Gastroenterology, Juntendo University School of Medicine, Tokyo, Japan

2 Department of Gastroenterology and Hepatology, Jikei University School of Medicine, Kashiwa Hospital, Kashiwa, Japan

3 Pathology and Cytology Laboratories, BML Inc. PCL Japan, Tokyo, Japan
Bibliography

Dol 10.1055/s-0030-1256451

Endoscopy 2011; 43: E298-E299

(c) Georg Thieme Verlag KG Stuttgart - New York . ISSN 0013-726X

\section{Corresponding author}

\section{T. Osada}

Department of Gastroenterology Juntendo University School of Medicine 2-1-1 Hongo

Bunkyo-ku

Tokyo 113-8421

Japan

Fax: +3-81-388621060

otaro@juntendo.ac.jp 